\title{
The Clark dual and multiple periodic solutions of delay differential equations
}

Huafeng Xiao*, Jianshe Yu and Zhiming Guo

* Correspondence: huafeng@gzhu. edu.cn

College of Mathematics and Information Sciences, Guangzhou University, Guangzhou, 510006, PRC

\section{Abstract}

We study the multiplicity of periodic solutions of a class of non-autonomous delay differential equations. By making full use of the Clark dual, the dual variational functional is considered. Some sufficient conditions are obtained to guarantee the existence of multiple periodic solutions.

2000 Mathematics Subject Classification: 34K13; 34K18.

Keywords: periodic solution, Clark dual, Morse-Ekeland index, delay differential equation, asymptotic linearity

\section{Introduction}

The existence and multiplicity of periodic solutions of delay differential equations have been investigated since 1962 . Various methods have been used to study such a problem [1-10]. Among those methods, critical point theory is a very important tool. By combining with Kaplan-Yorke method, it can be used indirectly to study the existence of periodic solutions of delay differential equation [11-16]. By building the variational frame for some special systems, it can be used directly to study the existence of delay differential systems $[17,18]$. However, the variational functionals in the above two cases are strongly indefinite. They are hard to be dealed with.

In this article, we study multiple periodic solutions of the following non-autonomous delay differential equations

$$
x^{\prime}(t)=-f\left(t, x\left(t-\frac{\pi}{2}\right)\right)
$$

where $x(t) \in \mathbb{R}^{n}, f \in C\left(\mathbb{R} \times \mathbb{R}^{n}, \mathbb{R}^{n}\right)$. We assume that

(f1) $f(t, x)$ is odd with respect to $x$ and $\pi / 2$-periodic with respect to $t$, i.e.,

$$
f(t,-x)=-f(t, x), \quad f\left(t+\frac{\pi}{2}, x\right)=f(t, x), \quad \forall(t, x) \in \mathbb{R} \times \mathbb{R}^{n} ;
$$

(f2) There exists a continuous differentiable function $F(t, x)$, which is strictly convex with respect to $x$ uniformly in $t$, such that $f(t, x)$ is the gradient of $F(t, x)$ with respect to $x$;

\section{Springer}

(C) 2011 Xiao et al; licensee Springer. This is an Open Access article distributed under the terms of the Creative Commons Attribution License (http://creativecommons.org/licenses/by/2.0), which permits unrestricted use, distribution, and reproduction in any medium, provided the original work is properly cited. 
(f3)

$$
\begin{aligned}
& f(t, x)=A_{0} x+g(t, x), \quad g(t, x)=o(|x|) \quad \text { as } \quad|x| \rightarrow 0 \quad \text { uniformly in } t \\
& f(t, x)=A_{\infty} x+h(t, x), \quad h(t, x)=o(|x|) \quad \text { as } \quad|x| \rightarrow \infty \quad \text { uniformly in } t ;
\end{aligned}
$$

where $A_{0}, A_{\infty}$ are positive definite constant matrices.

By making use of the Clarke dual, we study the dual variational functional associated with (1), which is an indefinite functional. Since the dimension of its negative space is finite, we define it as the Morse index of the dual variational functional. This Morse index is significant. Then $Z_{2}$-index theory can be used and some sufficient conditions are obtained to guarantee the existence of multiple periodic solutions of (1).

The rest of this article is organized as follows: in Section 2, some preliminary results will be stated; in Section 3, linear system is discussed and the Morse index of the variational functional associated with linear system is defined; in Section 4, our main results will be stated and proved.

\section{Preliminaries}

Denote by $\mathbb{N}, \mathbb{N} * \mathbb{Z}, \mathbb{R}$ the sets of all positive integers, nonnegative integers, integers, real numbers, respectively. We define $S^{1}:=\mathbb{R} /(2 \pi \mathbb{Z})$.

For a matrix $A$, denote by $\sigma(A)$ the set of eigenvalue of $A$. The identity matrix of order $n$ is denoted by $I_{n}$ and for simplicity by $I$.

Let $x, y \in L^{2}\left(S^{1}, \mathbb{R}^{n}\right)$. For every $z \in C^{\infty}\left(S^{1}, \mathbb{R}^{n}\right)$, if

$$
\int_{0}^{2 \pi}\left(x(t), z^{\prime}(t)\right) d t=-\int_{0}^{2 \pi}(\gamma(t), z(t)) d t
$$

then $y$ is called a weak derivative of $x$, denoted by $\dot{x}$.

The space $H^{1}=W^{1,2}\left(S^{1}, \mathbb{R}^{n}\right)$ consists of $2 \pi$-periodic vector-valued functions with dimension $n$, which possess square integrable derivative of order 1 . We can choose the usual norm and inner product in $H^{1}$ as follows:

$$
\begin{aligned}
& \|x\|^{2}=\int_{0}^{2 \pi}\left[|x(t)|^{2}+|\dot{x}(t)|^{2}\right] d t \\
& <x, y>=\int_{0}^{2 \pi}[(x(t), y(t))+(\dot{x}(t), \dot{y}(t))] d t, \quad \forall x, y \in H^{1},
\end{aligned}
$$

where $|\cdot|,(\cdot, \cdot)$ denote the usual norm and inner product in $\mathbb{R}^{n}$, respectively. Then $H^{1}$ is a Hilbert space.

Define the shift operator $K: H^{1} \rightarrow H^{1}$ by $K x(\cdot)=x(\cdot+\pi / 2)$, for all $x \in H^{1}$. Clearly, $K$ is a bounded linear operator from $H^{1}$ to $H^{1}$. Set

$$
E=\left\{x \in H^{1} \mid K^{2} x=-x\right\}
$$

Then $E$ is a closed subspace of $H^{1}$. If $x \in E$, its Fourier expansion is

$$
x(t)=\sum_{k=1}^{\infty}\left[a_{k} \cos (2 k-1) t+b_{k} \sin (2 k-1) t\right],
$$


where $a_{k}, b_{k} \in \mathbb{R}^{n}$. In particular, $E$ does not contain $\mathbb{R}^{n}$ as its subspace. In addition, $\int_{0}^{2 \pi} x(t) d t=0$ for all $x \in E$.

The dual variational functional corresponding to (1) defined on $H^{1}$ is

$$
J(\gamma)=\int_{0}^{2 \pi}\left[\frac{1}{2}\left(\dot{\gamma}\left(t+\frac{\pi}{2}\right), \gamma(t)\right)+F^{*}(t, \dot{\gamma}(t))\right] d t .
$$

By Hypothesis (f3), $F(t, x) /|x| \rightarrow+\infty$ as $|x| \rightarrow \infty$ uniformly in $t$. Since $F(t, \cdot)$ is strictly convex, Proposition 2.4 of [19] implies that $F^{*}(t, \cdot) \in C^{1}\left(\mathbb{R}^{n}, \mathbb{R}\right)$. Since $f$ satisfies (f3), it follows the discussion in Chapter 7 of [19] that

$$
\begin{aligned}
& f^{*}(t, y)=B_{\infty} y+o(|y|) \quad \text { as } \quad|y| \rightarrow \infty \quad \text { uniformly in } t \\
& f^{*}(t, y)=B_{0} y+o(|y|) \quad \text { as } \quad|y| \rightarrow 0 \quad \text { uniformly in } t
\end{aligned}
$$

where $B_{\infty}=A_{\infty}^{-1}$ and $B_{0}=A_{0}^{-1}$.

Lemma 2.1. If $y \in E$ is a critical point of $J$, then the function $x$ defined by $x(t)=f^{*}(t, \dot{\gamma}(t))$ is a $2 \pi$-periodic solution of $(1)$.

Proof. Since $f(\cdot, x)$ is $\pi / 2$-periodic, it follows that $F(\cdot, x)$ and then $F^{*}(\cdot, \dot{\gamma})$ are $\pi / 2$-periodic. So is $f^{*}(\cdot, \dot{y})$. (4) implies that there exist positive constants $a_{1}, a_{2}$ such that $\mid F^{*}(t$, $y)\left.\left|\leq a_{1}+a_{2}\right| y\right|^{s}$ for some $s>2$ and all $t \in \mathbb{R}, y \in H^{1}$. We define $\phi$ by the formulas

$$
\varphi(\gamma)=\int_{0}^{2 \pi} F^{*}(t, \dot{\gamma}(t)) d t
$$

It follows Proposition B. 37 of [20] that $\phi \in C^{1}\left(H^{1}, \mathbb{R}\right)$ and

$$
<\varphi^{\prime}(y), z>=\int_{0}^{2 \pi}\left(f^{*}(t, \dot{y}(t)), \dot{z}(t)\right) d t, \quad \forall y, z \in H^{1} .
$$

We claim: $\phi^{\prime}(y) \in E$ if $y \in E$.

To prove the above claim, let $z \in H^{1}$ and $y \in E$,

$$
\begin{aligned}
<K^{2} \varphi^{\prime}(y), z>= & <\varphi^{\prime}(y), K^{-2} z>=\int_{0}^{2 \pi}\left(f^{*}(t, \dot{\gamma}(t)), \dot{z}(t-\pi)\right) d t \\
& =\int_{0}^{2 \pi}\left(f^{*}(t+\pi, \dot{\gamma}(t+\pi)), \dot{z}(t)\right) d t=\int_{0}^{2 \pi}\left(f^{*}(t,-\dot{\gamma}(t)), \dot{z}(t)\right) d t \\
& =\int_{0}^{2 \pi}\left(-f^{*}(t, \dot{\gamma}(t)), \dot{z}(t)\right) d t=<-\varphi^{\prime}(\gamma), z>.
\end{aligned}
$$

The arbitrary of $z$ implies that $\phi^{\prime}(y) \in E$.

Since $\phi \in C^{1}\left(H^{1}, \mathbb{R}\right)$, it is easy to check that $J \in C^{1}\left(H^{1}, \mathbb{R}\right)$ and

$$
<J^{\prime}(\gamma), h>=\int_{0}^{2 \pi}\left(\frac{1}{2} \gamma\left(t-\frac{\pi}{2}\right)-\frac{1}{2} y\left(t+\frac{\pi}{2}\right)+f^{*}(t, \dot{\gamma}(t)), \dot{h}(t)\right) d t, \quad h \in H^{1} .
$$

Assume that $y \in E$ is a critical point of $J$. For any $h \in H^{1}, h=h_{1}+h_{2}$, where $h_{1} \in E$, $h_{2} \in E^{\perp}$. Then

$$
<J^{\prime}(y), h>=<J^{\prime}(y), h_{1}>+<J^{\prime}(y), h_{2}>.
$$


Since $\phi^{\prime}(y) \in E$, it is easy to check that $J^{\prime}(y) \in E$ and $\left\langle J^{\prime}(y), h_{2}>=0\right.$. Since $y$ is a critical point of $J$ on $E$, then $\left\langle J^{\prime}(y), h_{1}>=0\right.$. Thus $y$ is a critical point of $J$ on $H^{1}$. Applying the fundamental Lemma (cf. [19]), there exists $c_{1}$ such that

$$
y\left(t-\frac{\pi}{2}\right)+f^{*}(t, \dot{\gamma}(t))=c_{1}, \quad \text { a.e. on }[0,2 \pi]
$$

Setting

$$
x(t)=f^{*}(t, \dot{y}(t))=-\gamma\left(t-\frac{\pi}{2}\right)+c_{1}
$$

we obtain $x \in H^{1}, \dot{x}(t-\pi / 2)=\dot{y}(t)$ and by duality

$$
\dot{\gamma}(t)=f(t, x(t)) .
$$

Thus,

$$
\dot{x}\left(t-\frac{\pi}{2}\right)=f(t, x(t))
$$

i.e.,

$$
\dot{x}(t)=-f\left(t, x\left(t-\frac{\pi}{2}\right)\right) \quad \text { a.e. on }[0,2 \pi] .
$$

Moreover, $x(0)=x(2 \pi)$ since $x \in H^{1}$. It follows a regular discussion that $x(t)=f^{*}(t, \dot{y}(t))$ is a periodic solution of $(1)$.

Let $X$ be a Hilbert space, $\Phi \in C^{1}(X, \mathbb{R})$, i.e., $\Phi$ is a continuously Fréchet differentiable functional defined on $X$. If $X_{1}$ is a closed subspace of $X$, denote by $X_{1}^{\perp}$ the orthogonal complement of $X_{1}$ in $X$. Fix a prime integer $p>1$. Define a map $\mu: X \rightarrow X$ such that $\|\mu x\|=\|x\|$ for any $x \in X$ and $\mu^{p}=i d_{X}$, where $i d_{X}$ is the identity map on $X$. Then $\mu$ is a linear isometric action of $Z_{p}$ on $X$, where $Z_{p}$ is the cyclic group with order $p$.

A subset $A \subset X$ is called $\mu$-invariant if $\mu(A) \subset A$. A continuous map $h: A \rightarrow E$ is called $\mu$-equivariant if $h(\mu x)=\mu h(x)$ for any $x \in A$. A continuous functional $H: X \rightarrow$ $\mathbb{R}$ is called $\mu$-invariant if $H(\mu x)=H(x)$ for any $x \in X$.

$\Phi$ is said to be satisfying (PS)-condition if any sequence $\left\{x_{j}\right\} \subset X$ for which $\left\{\Phi\left(x_{j}\right)\right\}$ is bounded and $\Phi^{\prime}\left(x_{j}\right) \rightarrow 0$ as $j \rightarrow \infty$, possesses a convergent subsequence. A sequence $\left\{x_{j}\right\}$ is called (PS)-sequence if $\left\{\Phi\left(x_{j}\right)\right\}$ is bounded and $\Phi^{\prime}\left(x_{j}\right) \rightarrow 0$ as $j \rightarrow \infty$.

Lemma 2.2 [21]. Let $\Phi \in C^{1}(X, \mathbb{R})$ be a $\mu$-invariant functional satisfying the (PS)condition. Let $Y$ and $Z$ be closed $\mu$-invariant subspaces of $X$ with $\operatorname{codim} Y$ and $\operatorname{dim} Z$ finite and

$$
\text { codim } Y<\operatorname{dim} Z \text {. }
$$

Assume that the following conditions are satisfied:

(F1) $F i x_{\mu} \subset Y, Z \cap F i x_{\mu}=\{0\}$;

$(F 2) \inf _{x \in Y} \Phi(x)>-\infty$;

(F3) there exist constants $r>0$ and $c<0$ such that $\Phi(x) \leq c$ whenever $x \in Z$ and $\|$ $x \|=r$;

(F4) if $x \in$ Fix $x_{\mu}$ and $\Phi^{\prime}(x)=0$, then $\Phi(x) \geq 0$.

Then there exists at least $\operatorname{dim} Z-\operatorname{codim} Y$ distinct $Z_{p}$-orbits of critical points of $\Phi$ outside of Fix $x_{\mu}$ with critical value less than or equal to $c$. 


\section{Morse index}

Let $A$ be a positive definite constant matrix. We consider the periodic boundary value problem

$$
\left\{\begin{array}{l}
x^{\prime}(t)+A x\left(t-\frac{\pi}{2}\right)=0 \\
x(0)=x(2 \pi)
\end{array} .\right.
$$

Since $F(t, x)=1 / 2(A x, x)$, it is easy to verify that its Legendre transform $F^{*}(t, y)$ is of the form $F^{*}(t, y)=1 / 2(B y, y)$, where $B=A^{-1}$. The dual action of (6) is defined on $H^{1}$ by

$$
\chi_{A}(\gamma)=\int_{0}^{2 \pi} \frac{1}{2}\left[\left(\dot{\gamma}\left(t+\frac{\pi}{2}\right), \gamma(t)\right)+(B \dot{\gamma}(t), \dot{\gamma}(t))\right] d t .
$$

$A$ is positively definite, so is $B$. Thus there exists $\delta_{1}>0$ such that $(B y, y) \geq \delta_{1}|y|^{2}$ for all $y \in \mathbb{R}^{n}$. Wirtinger's inequality implies that the symmetric bilinear form given by

$$
((y, z))_{1}=\int_{0}^{2 \pi}(B \dot{\gamma}(t), \dot{z}(t)) d t
$$

define an inner product on $E$. The corresponding norm $\|\cdot\|_{1}$ is such that

$$
\|y\|_{1}^{2} \geq \delta_{1}\|\dot{y}\|_{L^{2}}^{2} .
$$

Proposition 3.1. The norm $\|\cdot\|_{1}$ is equivariant to the stand norm $\|\cdot\|$ of $E$.

Proof. Since $B$ is positive, Wirtinger's inequality and (9) imply that there exists a positive constant $\delta_{2}$ such that

$$
\|x\|_{1}^{2}=\int_{0}^{2 \pi}(B \dot{x}(t), \dot{x}(t)) d t \geq \delta_{1}\|\dot{x}\|_{L^{2}}^{2} \geq \delta_{1} \delta_{2} \| x||^{2} .
$$

On the other hand, since $B$ is a positive definite matrix, there exists a constant $M>0$ such that

$$
\|x\|^{2} \geq\|\dot{x}\|_{L^{2}}^{2} \geq \frac{1}{M} \int_{0}^{2 \pi}(B \dot{x}(t), \dot{x}(t)) d t=\frac{1}{M}\|x\|_{1}^{2} .
$$

Thus those two norms are equivariant to each other, which completes our proof.

Let us define the linear operator $L$ on $E$ by setting

$$
((L y, z))_{1}=\int_{0}^{2 \pi}\left(y\left(t+\frac{\pi}{2}\right), \dot{z}(t)\right) d t .
$$

One can easily check that $L$ is a compact self-adjoint operator. (7) can be rewritten as

$$
2 \chi_{A}(y)=\int_{0}^{2 \pi}\left[\left(\dot{\gamma}\left(t+\frac{\pi}{2}\right), \gamma(t)\right)+(B \dot{y}(t), \dot{\gamma}(t))\right] d t=(((I-L) y, y))_{1} .
$$

It follows from the spectral theory that $E$ can be decomposed as the orthogonal sum of $\operatorname{Ker}(I-L), E^{+}$and $E^{-}$with $I-L$ positive definite (resp. negative definite) on $E^{+}$(resp. $E^{-}$). Since $L$ is compact, it has at most finite many eigenvalues (counting the multiplicity) greater than one. Thus the index $\operatorname{dim} E^{-}<\infty$. 
Definition 3.1. The index $i(A)$ is the Morse index of $\chi_{A}$, i.e. the supermum of the dimension of the subspace of $E$ on which $\chi_{A}$ is negative definite.

On the other hand, there exists a positive constant $\delta_{3}$ such that

$$
(((I-L) y, y))_{1} \geq \delta_{3}\|y\|_{1}^{2}, \forall y \in E^{+} \quad \text { and } \quad(((I-L) y, y))_{1} \leq-\delta_{3}\|y\|_{1}^{2}, \quad \forall y \in E^{-} .
$$

Setting $\delta=\delta_{1} \delta_{3}>0$, we reduce from (9) and (11) the estimates

$$
\chi_{A}(y) \geq \frac{\delta}{2}\|\dot{y}\|_{L^{2}}^{2}, \quad \forall y \in E^{+} \quad \text { and } \quad \chi_{A}(y) \leq-\frac{\delta}{2}\|\dot{y}\|_{L^{2}}^{2}, \quad \forall y \in E^{-} .
$$

Proposition 3.2. The dimension of $\operatorname{Ker}(I-L)$ is equal to the number of linearly independent solutions of (6).

Proof. If $y \in E$, by a Fourier argument, $y \in \operatorname{Ker}(I-L)$ if and only if

$$
B \dot{y}(t)=\gamma\left(t+\frac{\pi}{2}\right)+c_{2}
$$

for some $c_{2} \in \mathbb{R}^{n}$ and a.e. $t \in[0,2 \pi]$. Since $B=A^{-1}$ is invertible, $y$ is continuous differentiable. Thus (13) hold for all $t \in[0,2 \pi]$, and $B \dot{y} \in E \oplus \mathbb{R}^{n}$. Set

$$
x(t)=(\varphi y)(t)=B \dot{y}(t) .
$$

In view of (13), (14) and the Clark dual, we have

$$
\dot{x}(t)=\frac{d}{d t}(B \dot{y}(t))=\dot{y}\left(t+\frac{\pi}{2}\right)=A x\left(t+\frac{\pi}{2}\right) .
$$

Thus $x$ is a solution of (6).

Conversely, assume now that $x \in E \oplus \mathbb{R}^{n}$ is a solution of (6). Then

$$
\int_{0}^{2 \pi} A x\left(t-\frac{\pi}{2}\right) d t=0,
$$

and hence there exists an unique $y \in E$ such that $x=\phi y$, where $\phi$ is defined by (14).

Thus

$$
-\dot{x}(t)=A x\left(t-\frac{\pi}{2}\right)=\dot{y}\left(t-\frac{\pi}{2}\right)
$$

for a.e. $t \in[0,2 \pi]$. Integrating the above equality, we have $x(t)=y(t+\pi / 2)+c_{3}$ for some $c_{3} \in \mathbb{R}^{n}$ and all $t \in[0,2 \pi]$. Consequently,

$$
A\left[y\left(t+\frac{\pi}{2}\right)+c_{3}\right]=\dot{\gamma}(t)
$$

for a.e. $t \in[0,2 \pi]$, which is equivariant to (13) and shows that $y \in \operatorname{Ker}(I-L)$. Thus $\phi$ is an isomorphism between $\operatorname{Ker}(I-L)$ and the space of solutions of (6).

Consider the following eigenvalue problem

$$
(I-L) y=\lambda y, \quad \forall y \in E .
$$

For any $z \in E$, we have $(((I-L) y, z))_{1}=>\lambda((y, z))_{1}$. Computing directly, we have

$$
\begin{gathered}
\int_{0}^{2 \pi}\left(\sum_{k=1}^{\infty}\left[(2 k-1) B+(-1)^{k} I\right]\left[-a_{k} \cos (2 k-1) t+b_{k} \sin (2 k-1) t\right], \dot{z}(t)\right) d t \\
=\lambda \int_{0}^{2 \pi}\left(\sum_{k=1}^{\infty}(2 k-1) B\left[-a_{k} \cos (2 k-1) t+b_{k} \sin (2 k-1) t\right], \dot{z}(t)\right) d t
\end{gathered}
$$


Denote by $e_{i}, i=1,2, \ldots, n$ the basic of $\mathbb{R}^{n}$. Choosing

$$
z(t)=\left\{\begin{array}{l}
\sin (2 k-1) t \\
\cos (2 k-1) t
\end{array}\right\} e_{i}, \quad k \in \mathbb{N}, i=1,2, \ldots, n,
$$

it follows that

$$
\left\{\begin{array}{l}
\left((2 k-1) B+(-1)^{k} I\right) a_{k}=\lambda(2 k-1) B a_{k} \\
\left((2 k-1) B+(-1)^{k} I\right) b_{k}=\lambda(2 k-1) B b_{k} .
\end{array}\right.
$$

Since $B=A^{-1}$, then

$$
\left\{\begin{array}{l}
\lambda a_{k}=\left(\frac{(-1)^{k}}{2 k-1} A+I\right) a_{k} \\
\lambda b_{k}=\left(\frac{(-1)^{k}}{2 k-1} A+I\right) b_{k} .
\end{array}\right.
$$

Thus

$$
\operatorname{dim} \operatorname{Ker}(I-L)=2 \sum_{k=1}^{\infty} \operatorname{dim} \operatorname{Ker}\left(\frac{(-1)^{k}}{2 k-1} A+I\right)
$$

Proposition 3.3. If $\sigma(A) \cap\{4 l+1, l \in \mathbb{N} *\}=\varnothing$, then $I-L$ is invertible and codim $E^{+}$ $=i(A)$.

Proof. The above analysis implies that $\operatorname{Ker}(I-L)=\{0\}$ if $\sigma(A) \cap\left\{4 l+1, l \in \mathbb{N}^{*}\right\}=\varnothing$. Thus $I-L$ is invertible. If we decompose $E$ as $E=E^{+} \oplus E^{-}$, then $\operatorname{codim} E^{+}=i(A)$.

\section{Main results and their proofs}

Now we consider the multiplicity of periodic solutions of (1). The main result reads as follows:

Theorem 4.1. Assume that (f1)-(f3) are satisfied. Moreover,

(A1) $\sigma\left(A_{\infty}\right) \cap\{4 l+1, l \in \mathbb{N} *\}=\varnothing$;

(A2) $i\left(A_{0}\right)>i\left(A_{\infty}\right)$.

Then (1) has at least $i\left(A_{0}\right)-i\left(A_{\infty}\right)$ pairs of nontrivial $2 \pi$-periodic solutions.

By (f3), $f(t, 0)=0$ uniformly in $t$. Since $F(t, \cdot)$ is strictly convex, it follows that 0 is the unique equilibrium point of (1). Without loss of generality, we can assume that $F$ $(t, 0)=0$ uniformly in $t$. Since $f(t, 0)=0$, then $F^{*}(t, 0)=0$ uniformly in $t$.

Lemma 4.1. The functional J satisfies (PS)-condition.

Proof. Denote by $((\cdot, \cdot))_{1, \infty}$ the inner product, defined by (8) replacing $B$ by $B_{\infty}$. Let $L_{\infty}$ be linear self-adjoint operator, defined by $(10)$, under the inner product $((\cdot, \cdot))_{1, \infty}$. We define the operator $N$ over $E$ by setting

$$
((N y, z))_{1, \infty}=\int_{0}^{2 \pi}\left(f^{*}(t, \dot{y})-B_{\infty} \dot{y}, \dot{z}\right) d t, \quad \forall y, z \in E .
$$

Let $\left\{y_{j}\right\}_{j=1}^{\infty}$ be a (PS)-sequence. Define $f_{j}:=y_{j}-L_{\infty} y_{j}+N y_{j}, j \in \mathbb{N}$. Since

$$
<J^{\prime}(\gamma), z>=\int_{0}^{2 \pi}\left(y\left(t-\frac{\pi}{2}\right)+f^{*}(t, \dot{\gamma}), \dot{z}\right) d t=\left(\left(y-L_{\infty} \gamma+N y, z\right)\right)_{1, \infty}
$$


then $f_{j} \rightarrow 0$ in $E$ as $j \rightarrow \infty$. Then there exists $R>0$ such that for all $j \in \mathbb{N},\left\|f_{j}\right\| \leq R$. (A1) implies that $M=I-L_{\infty}$ is invertible. Thus it follows from (4) that there exists a positive constant $c_{4}$ such that, for all $y \in E$

$$
\left\|N y||_{1, \infty} \leq \frac{1}{2}\right\| M^{-1}\left\|_{1, \infty}^{-1}\right\| y||_{1, \infty}+c_{4}
$$

where $\|\cdot\|_{1, \infty}$ denotes by the norm corresponding to $((\cdot, \cdot))_{1, \infty}$. Since $\left\|f_{j}\right\| \leq R$, we have

$$
\left\|y_{j}||_{1, \infty} \leq\right\| M^{-1}||_{1, \infty}\left(\left\|N y_{j}||_{1, \infty}+\right\| f_{j}||_{1, \infty}\right) \leq \frac{1}{2}\left\|y_{j}||_{1, \infty}+\right\| M^{-1}||_{1, \infty}\left(c_{4}+R\right), \quad j \in \mathbb{N} .
$$

The above inequality implies that $\left\{y_{j}\right\}$ is bounded. A standard argument as Lemma 4.5 in [19] shows that $\left\{y_{j}\right\}$ has a convergent sequence.

Lemma 4.2. The functional $J$ is bounded from below on a closed invariant subspace $Y$ of $E$ of codimension $i\left(A_{\infty}\right)$.

Proof. Consider the linear delay differential system

$$
x^{\prime}(t)=-A_{\infty} x\left(t-\frac{\pi}{2}\right) .
$$

Its dual variational functional is

$$
\chi_{A_{\infty}}(y)=\int_{0}^{2 \pi} \frac{1}{2}\left[\left(\dot{\gamma}\left(t+\frac{\pi}{2}\right), \gamma(t)\right)+\left(B_{\infty} \dot{y}(t), \dot{\gamma}(t)\right)\right] d t,
$$

where $((\cdot, \cdot))_{1, \infty}, L_{\infty}$ are defined as Lemma 4.1.

Because of (A1) and Proposition 3.3, the operator $I-L_{\infty}$ is invertible. We decompose $E$ as $E=E_{\infty}^{+} \oplus E_{\infty}^{-}$, where $E_{\infty}^{+}$(resp. $E_{\infty}^{-}$) is the positive (resp. negative) definite eigenspace of $I-L_{\infty}$. Then $\operatorname{codim} E_{\infty}^{+}=\operatorname{dim} E_{\infty}^{-}=i\left(A_{\infty}\right)$. Let $Y:=E_{\infty}^{+}$. Inequality (12) implies that there exists a positive constant $\delta_{4}$ such that, for each $y \in Y$,

$$
\left.\chi_{A_{\infty}}(\gamma)=\int_{0}^{2 \pi} \frac{1}{2}\left[\dot{\gamma}\left(t+\frac{\pi}{2}\right), \gamma(t)\right)+\left(B_{\infty} \dot{\gamma}, \dot{\gamma}\right)\right] d t \geq \frac{\delta_{4}}{2}\|\dot{\gamma}\|_{L^{2}}^{2} .
$$

It follows (4) that there exists a positive constant $c_{5}$ such that

$$
\left|f^{*}(t, y)-B_{\infty} y\right| \leq \frac{\delta_{4}}{2}|y|+c_{5}
$$

for each $y \in \mathbb{R}^{n}$. Hence, by the mean value theorem,

$$
\begin{aligned}
\left|F^{*}(t, y)-\frac{1}{2}\left(B_{\infty} y, y\right)\right| \leq \int_{0}^{1} \mid & \left(f^{*}(t, \sigma y)-\sigma B_{\infty} y, y\right) \mid d \sigma \\
\leq & \int_{0}^{1}\left[\frac{\delta_{4}}{2} \sigma|y|^{2}+c_{5}|y|\right] d \sigma=\frac{\delta_{4}}{4}|y|^{2}+c_{5}|y| .
\end{aligned}
$$

Consequently, we have, for $y \in Y$,

$$
\begin{aligned}
J(y) & =\chi_{A_{\infty}}(y)+\int_{0}^{2 \pi}\left[F^{*}(t, \dot{y})-\frac{1}{2}\left(B_{\infty} \dot{y}, \dot{y}\right)\right] d t \\
& \geq \frac{\delta_{4}}{2}\|\dot{y}\|_{L^{2}}^{2}-\int_{0}^{2 \pi}\left[\frac{\delta_{4}}{4}|\dot{y}|^{2}+c_{5}|\dot{y}|\right] d t \\
& =\frac{\delta_{4}}{4}\|\dot{y}\|_{L^{2}}^{2}-c_{5}\left\|\dot{y}||_{L^{1}} \geq \frac{\delta_{4}}{4}\right\| \dot{y}\left\|_{L^{2}}^{2}-c_{5} \sqrt{2 \pi}\right\| \dot{y}||_{L^{2}}
\end{aligned}
$$

and $J$ is bounded from below on $Y$. 
Lemma 4.3. There exists an invariant subspace $Z$ of $E$ with dimension $i\left(A_{0}\right)$ and some $r>0$ such that $J(y)<0$ whenever $y \in Z$ and $\|y\|_{1}=r$.

Proof. Consider the linear delay differential system

$$
x^{\prime}(t)=-A_{0} x\left(t-\frac{\pi}{2}\right) .
$$

Its dual variational functional is

$$
\chi_{A_{0}}(\gamma)=\int_{0}^{2 \pi} \frac{1}{2}\left[\left(\dot{\gamma}\left(t+\frac{\pi}{2}\right), \gamma(t)\right)+\left(B_{0} \dot{y}(t), \dot{\gamma}(t)\right)\right] d t
$$

where $((\cdot, \cdot))_{1,0}, L_{0}$ are defined by (8) and (10) with $B$ replaced by $B_{0}$, respectively.

We decompose $E$ as $E=E_{0}^{+} \oplus E_{0}^{0} \oplus E_{0}^{-}$, where $E_{0}^{0}=\operatorname{ker}\left(I-L_{0}\right)$, $E_{0}^{+}$(resp. $\left.E_{0}^{-}\right)$is the positive (resp. negative) definite eigenspace of $I-L_{0}$. Let $Z:=E_{0}^{-}$. Then $Z$ is a finite dimensional space. Inequality (12) implies that there exists $\delta_{5}>0$ such that, for each $y$ $\in Z$,

$$
\chi_{A_{0}}(\gamma)=\int_{0}^{2 \pi} \frac{1}{2}\left[\left(\dot{\gamma}\left(t+\frac{\pi}{2}\right), \gamma(t)\right)+\left(B_{0} \dot{y}, \dot{\gamma}\right)\right] d t \leq-\frac{\delta_{5}}{2}\|\dot{\gamma}\|_{L^{2}}^{2}
$$

whenever $y \in Z$. By (5), there exists $\rho>0$ such that

$$
\left|f^{*}(t, y)-B_{0} y\right| \leq \frac{\delta_{5}}{2}|y|
$$

for $y \in \mathbb{R}^{n}$ with $|y| \leq \rho$. Hence, by the mean value theorem, we have

$$
\begin{aligned}
\left|F^{*}(t, y)-\frac{1}{2}\left(B_{0} y, y\right)\right| \leq & \int_{0}^{1}\left|\left(f^{*}(t, \sigma y)-\sigma B_{0} y, y\right)\right| d \sigma \\
& \leq \int_{0}^{1} \frac{\delta_{5}}{2} \sigma|y|^{2} d \sigma=\frac{\delta_{5}}{4}|y|^{2}
\end{aligned}
$$

whenever $|y| \leq \rho$. Consequently, if $y \in Z$ and $0<|y|<\rho$, we get

$$
\begin{aligned}
J(y) & =\chi_{A_{0}}(\gamma)+\int_{0}^{2 \pi}\left[F^{*}(t, \dot{\gamma})-\frac{1}{2}\left(B_{0} \dot{\gamma}, \dot{\gamma}\right)\right] d t \\
& \leq-\frac{\delta_{5}}{2}\|\dot{\gamma}\|_{L^{2}}^{2}+\int_{0}^{2 \pi} \frac{\delta_{5}}{4}|\dot{y}|^{2} d t=-\frac{\delta_{5}}{4}\|\dot{y}\|_{L^{2}}^{2} \leq 0 .
\end{aligned}
$$

If $J(y)=0$, then $\| \dot{y}||_{L^{2}}=0$, which implies that $|\dot{y}|=0$ for a.e. $t \in[0,2 \pi]$. Thus $y$ $\in \mathbb{R}^{n} \cap Z=\{0\}$, which contradicts with $0<|y|<\rho$. Thus $J(y)<0$.

Proof of Theorem 4.1. Now, we apply Lemma 2.2 to prove Theorem 4.1.

Define the action $\mu: E \rightarrow E$ by $\mu x=-x$. Then $\mu$ is a generator of group $Z_{2}$ and $\operatorname{Fix}_{\mu}$ $=\{0\}$. Obviously, (F1) and (F4) hold. It is easy to check that $J$ is $\mu$-invariant. Lemma 4.1 implies that $J$ satisfies (PS)-condition.

Let $Y, Z$ define as in Lemma 4.2 and 4.3. Then $Y, Z$ are $\mu$-invariant subspaces and (A2) implies that

$$
\operatorname{dim} Z=i\left(A_{0}\right)>i\left(A_{\infty}\right)=\operatorname{codim} Y .
$$

It follows from Lemmas 4.2 and 4.3 that (F2) and (F3) hold. Applying Lemma 2.2, J has at least $i\left(A_{0}\right)-i\left(A_{\infty}\right)$ pairs of distinct critical points with critical values less than or 
equal to $c$. Since $J(0)=0$ and $E \cap \mathbb{R}^{n}=\{0\}$, if follows that all those $i\left(A_{0}\right)-i\left(A_{\infty}\right)$ pairs of distinct critical points are nonconstant.

If $y$ is a critical point of $J$, then $x:=f^{*}(t, \dot{y})$ is a $2 \pi$-periodic solution of (1). Clearly, $y$ $\neq 0$ implies that $x \neq 0$. Let $x_{1}, x_{2}$ are two periodic solutions satisfying $x_{1}=-x_{2}$. Setting $\dot{y}_{1}=f\left(t, x_{1}\right)$ and $\dot{y}_{2}=f\left(t, x_{2}\right)$, then

$$
\dot{y}_{1}=f\left(t, x_{1}\right)=f\left(t,-x_{2}\right)=-f\left(t, x_{2}\right)=-\dot{y}_{2} .
$$

Integrating he above equality, we have $y_{1}=-y_{2}+c_{6}$ for some $c_{6} \in \mathbb{R}^{n}$. Since $y_{1}, y_{2} \in$ $E$, it follows that $c_{6}=0$. Then $y_{1}, y_{2}$ belongs the same $Z_{2}$-orbit. Thus (1) has $i\left(A_{0}\right)-i$ $\left(A_{\infty}\right)$ pairs of nontrivial periodic solutions.

Corollary 4.1. Under the hypotheses of Theorem 4.1, (1) has at least $2\left[i\left(A_{0}\right)-i\left(A_{\infty}\right)\right]$ nontrivial $2 \pi$-periodic solutions.

Since every $Z_{2}$-orbit has two elements and those two elements are different from each other, this corollary is obvious.

\section{Acknowledgements}

This project was supported by the National Natural Science Foundation of China (Nos. 11031002 and 10871053). The authors are very grateful to the anonymous referee whose careful reading of the manuscript and valuable comments enhanced presentation of the manuscript.

\section{Authors' contributions}

The manuscript was drafted by HX and it is based on his PhD thesis. JY and ZG were the supervisors of the thesis and gave detailed comments on the manuscript. All authors read and approved the final manuscript.

\section{Competing interests}

The authors declare that they have no competing interests.

Received: 18 May 2011 Accepted: 11 November 2011 Published: 11 November 2011

\section{References}

1. Chow, S, Mallet-Paret, J: The fuller index and global Hopf bifurcation. J Diff Eqn. 29, 66-85 (1978). doi:10.1016/00220396(78)90041-4

2. Grafton, R: A periodicity theorem for autonomous functional differential equations. J Diff Eqn. 6, 87-109 (1969). doi:10.1016/0022-0396(69)90119-3

3. Kaplan, J, Yorke, J: On the stability of a periodic solution of a differential delay equation. SIAM J Math Anal. 6, 268-282 (1975). doi:10.1137/0506028

4. Kaplan, J, Yorke, J: On the nonlinear differential delay equation $x^{\prime}(t)=-f(x(t), x(t-1))$. J Diff Eqn. 23, $293-314(1977)$. doi:10.1016/0022-0396(77)90132-2

5. Mallet-Paret, J, Sell, G: The Poincare-Bendixson theorem for monotone cyclic feedback systems with delay. J Diff Eqn. 125, 441-489 (1996). doi:10.1006/jdeq.1996.0037

6. Mawhin, J: Periodic solutions of nonlinear functional differential equations. J Diff Eqn. 10, $240-261$ (1971). doi:10.1016/ 0022-0396(71)90049-0

7. Mawhin, J: Equivalence theorems for nonlinear operator equations and coincidence degree theory for some mappings in locally convex topological vector space. J Diff Eqn. 12, 610-636 (1972). doi:10.1016/0022-0396(72)90028-9

8. Nussbaum, R: Periodic solutions of some nonlinear autonomous functional differential equations, II. J Diff Eqn. 14, 368-394 (1973)

9. Nussbaum, R: Periodic solutions of some nonlinear autonomous functional differential equations. Ann Math Pura Appl. 10, 263-306 (1974)

10. Nussbaum, R: A Hopf global bifurcation theorem for retarded functional differential equations. Trans Am Math Soc. 238, 139-163 (1978)

11. Fei, G: Multiple periodic solutions of differential delay equations via Hamiltonian systems (I). Nonlinear Anal. 65, 25-39 (2006). doi:10.1016/j.na.2005.06.011

12. Fei, G: Multiple periodic solutions of differential delay equations via Hamiltonian systems (II). Nonlinear Anal. 65, 40-58 (2006). doi:10.1016/j.na.2005.06.012

13. Kaplan, J, Yorke, J: Ordinary differential equations which yield periodic solution of delay equations. J Math Anal Appl. 48, 317-324 (1974). doi:10.1016/0022-247X(74)90162-0

14. Li, J, He, X: Multiple periodic solutions of differential delay equations created by asymptotically linear Hamiltonian systems. Nonlinear Anal. 31, 45-54 (1998). doi:10.1016/S0362-546X(96)00058-2

15. Li, J, He, X: Periodic solutions of some differential delay equations created by Hamiltonian systems. Bull Aust Math Soc. 60, 377-390 (1999). doi:10.1017/S000497270003656X

16. $\mathrm{Li}, \mathrm{J}, \mathrm{He}, \mathrm{X}$ : Proof and generalization of Kaplan-Yorke's conjecture on periodic solution of differential delay equations. Sci China (Ser A). 42, 957-964 (1999). doi:10.1007/BF02880387 
17. Guo, C, Guo, Z: Existence of multiple periodic solutions for a class of second-order delay differential equations. Nonlinear Anal. 10, 3285-3297 (2009). doi:10.1016/j.nonrwa.2008.10.023

18. Guo, Z, Yu, J: Multiplicity results for periodic solutions to delay differential equations via critical point theory. J Diff Eqn. 218, 15-35 (2005). doi:10.1016/j.jde.2005.08.007

19. Mawhin, J, Willem, M: Critical Point Theory and Hamiltonian Systems. Springer, New York (1989)

20. Rabinowitz, P: Minimax methods in critical point theory with applications to differential equations. CBMS Reg Conf Ser in Math, New York. (1986)

21. Guo, Z, Yu, J: Multiplicity results for periodic solutions to second-order difference equations. J Dyn Diff Eqn. 18, 943-960 (2006). doi:10.1007/s10884-006-9042-1

doi:10.1186/1687-2770-2011-44

Cite this article as: Xiao et al:: The Clark dual and multiple periodic solutions of delay differential equations.

Boundary Value Problems 2011 2011:44.

\section{Submit your manuscript to a SpringerOpen ${ }^{\circ}$} journal and benefit from:

- Convenient online submission

- Rigorous peer review

- Immediate publication on acceptance

- Open access: articles freely available online

- High visibility within the field

- Retaining the copyright to your article

Submit your next manuscript at $\boldsymbol{\nabla}$ springeropen.com 